\title{
ON THE SANDS AND REES MEASUREMENT METHOD OF THE LONGITUDINAL COUPLING IMPEDANCE
}

\author{
A. Argan, INFN- LNF; L. Palumbo ${ }^{+}$, Dip. di Energetica-Roma; \\ M.R. Masullo, INFN- Napoli; V.G. Vaccaro, INFN- Napoli/Dip. Sci. Fisiche
}

\section{Abstract}

By means of a general theory we examine the Sands and Rees method of measurement of the longitudinal coupling impedance between a particle beam and the vacuum chamber components. We discuss the validity limits of the method in relation to the presence of the central wire which simulates the beam.

\section{INTRODUCTION}

The longitudinal coupling impedance (LCI) of an ultrarelativistic point charge $q$, travelling on a beam pipe axis, is defined as:

$$
Z(\omega)=-\frac{1}{q} \int_{-\infty}^{+\infty} E_{s z}(r=0, z ; \omega) e^{j k} 0^{z} d z
$$

where $\vec{E}_{s}$ is the Fourier transform of the longitudinal electric field on the pipe axis and $k_{0}=\omega / c$. The impedance is often measured by means of the transmission Scattering (S) parameter of the device. This method has been proposed in 70's on the ground of intuitive considerations. The basic idea is that the relativistic beam fields in the vacuum chamber can be simulated by means of a TEM wave propagating thanks to the presence of a central wire $[1,2,3]$. Several formulae have been proposed to express the LCI as function of the transmission S-parameter. The first in order of time is the so called relation of Sands and Rees suggested for the estimate of $Z(\omega)$ when the wire radius is very small [4]:

$$
Z(\omega)=2 R_{0}\left(\frac{S_{2,1}^{R E F}-S_{2,1}^{D U T}}{S_{2,1}^{R E F}}\right)
$$

$S_{2,1}^{D U T}$ is the transmission parameter of the component under test, $S_{2,1}^{R E F}$ the parameter of a portion of unperturbed coaxial line of the same length and $R_{0}$ is the characteristic impedance of the transmission line.

An improved relation valid for a single lumped impedance has been provided by Hahn and Pedersen [5] where $S_{2,1}^{R E F}$ at the denominator of (2) has been replaced by $S_{2,1}^{D U T}$. Other expressions have been proposed for distributed impedances [6,7] for which the Hahn and Pedersen formula breaks down.

Aim of this paper is to give a more rigorous proof of the relation (2) when the wire radius approaches zero. All the results have been obtained using only the Schelkunoff's Field Equivalence Principle and the Lorentz Reciprocity Theorem.

\section{LONGITUDINAL COUPLING IMPEDANCE BELOW CUTOFF}

A point charge $q$ moving, with velocity $v= \pm c i_{z}$, along $z$ axis of a conducting beam pipe of radius $b$ has a current density spectrum given by:

$$
\vec{J}_{q}^{ \pm}(\vec{r}, z ; \omega)= \pm q \frac{\delta(r)}{2 \pi r} e^{\mp j k_{0} z} \hat{i}_{z}
$$

and produces electromagnetic fields $\vec{E}_{q}^{ \pm}$and $\vec{H}_{q}^{ \pm}$on the pipe walls given by:

$$
\begin{aligned}
& \vec{E}_{q}^{ \pm}(r=b, \varphi, z ; \omega)=Z_{0} \frac{q}{2 \pi b} e^{\mp j k_{0} z} \hat{i}_{r} \\
& \vec{H}_{q}^{ \pm}(r=b, \varphi, z ; \omega)= \pm \frac{q}{2 \pi b} e^{\mp j k_{0} z} \hat{i}_{\varphi}
\end{aligned}
$$

where $Z_{0}$ is the characteristic impedance of the vacuum.

Let the beam pipe have an aperture $S_{A}$ on the conducting wall which in general can couple to an external structure. By using the Schelkunoff's Field Equivalence Principle, we may consider a system of equivalent magnetic currents $\vec{J}_{m s}$ on the aperture surface $S_{A}$. The electromagnetic fields into the waveguide can be written as:

$$
\vec{E}=\vec{E}_{q}^{+}+\vec{E}_{s} \quad \vec{H}=\vec{H}_{q}^{+}+\vec{H}_{s}
$$

where $\vec{E}_{s}$ and $\vec{H}_{s}$ are the fields scattered by the aperture, i.e., the fields radiated by the surface currents $\vec{J}_{m s}$ inside the pipe.

In order to calculate the LCI as function of the magnetic currents $\vec{J}_{m s}$ [8,9] we can apply the Lorentz Reciprocity Theorem relating the fields $\vec{E}_{q}^{-}, \vec{H}_{q}^{-}$to the fields $\vec{E}_{s}, \vec{H}_{s}$ of the unperturbed structure. Below the cutoff frequency, for an infinite pipe, we get [10] :

$$
-\frac{1}{q} \int_{-\infty}^{+\infty} E_{s z}(r=0, z) e^{j k_{0} z} d z=-\frac{1}{q^{2}} \iint_{S_{A}} \vec{H}_{q}^{-} \cdot \vec{J}_{m s} d S
$$

which recalling the definition (1) becomes:

$$
Z(\omega)=\frac{1}{2 \pi q b} \iint_{S_{A}} J_{m s \varphi} e^{j k_{0} z} d S
$$




\section{BEAM SIMULATED BY A WIRE}

We assume, now, that in the same pipe a perfectly conducting wire of radius $a$ is streched along the $z$ axis. The beam pipe so modified becomes a transmission line. In this new configuration, we consider a generator which excites a TEM wave $\vec{E}_{q}^{\prime+}, \vec{H}_{q}^{\prime+}$ reproducing the field $\vec{E}_{q}^{+}, \vec{H}_{q}^{+}$ of the charge in the unperturbed waveguide. We have, then:

$$
\vec{E}_{q}^{\prime+}=\frac{q Z_{0}}{2 \pi r} e^{-j k_{0} z} \hat{i}_{r} \quad \vec{H}_{q}^{\prime+}=\frac{q}{2 \pi r} e^{-j k_{0} z} \hat{i}_{\varphi}
$$

Calling, now, $\vec{E}_{A}^{\prime}$ the electric field on the aperture and $\vec{J}_{m s}^{\prime}$ the equivalent magnetic surface currents, we have that:

$$
\vec{J}_{m s}^{\prime}=\hat{i}_{r} \times \vec{E}_{A}^{\prime}
$$

The electromagnetic field inside the coaxial line can be expressed by the relations:

$$
\vec{E}^{\prime}=\vec{E}_{q}^{\prime+}+\vec{E}_{s}^{\prime} \quad \vec{H}^{\prime}=\vec{H}_{q}^{\prime+}+\vec{H}_{s}^{\prime}
$$

where $\vec{E}_{s}^{\prime}$ and $\vec{H}_{s}^{\prime}$ are the fields scattered by the aperture.

Below the cutoff frequency of the coaxial line mode $T E_{1,1}$, the fields $\vec{E}_{s}^{\prime}$ and $\vec{H}_{s}^{\prime}$ at a sufficient distance from the aperture can be represented by means of the TEM components only:

$$
\vec{E}_{s}^{\prime \pm}=\frac{\alpha^{ \pm}}{r} e^{\mp j k_{0} z} \hat{i}_{r} \quad \vec{H}_{s}^{\prime \pm}= \pm \frac{\alpha^{ \pm}}{Z_{0} r} e^{\mp j k_{0} z} \hat{i}_{\varphi}
$$

In order to calculate the coefficients $\alpha^{+}$and $\alpha^{-}$we apply the Lorentz Reciprocity Theorem to the volume $\mathrm{V}_{1}$ (fig.1), getting:

$$
\alpha^{ \pm}=\mp \frac{1}{4 \pi b \ln \left(\frac{b}{a}\right)} \iint_{S_{A}} J_{m s \varphi}^{\prime} e^{ \pm j k_{0} z} d S
$$

We observe that the TEM components of the fields scattered by the aperture vanish as the wire radius $a$ tends to zero. Furthermore the eigenfunctions of the coaxial line approach the cylindrical waveguide modes for $a \rightarrow 0$, therefore, the surface currents $\vec{J}_{m s}^{\prime}$ tend to the $\vec{J}_{m s}$. Thus we can express the longitudinal coupling impedance:

$$
\lim _{a \rightarrow 0} Z(\omega)=\frac{1}{2 \pi q b} \iint_{S_{A}} J_{m s \varphi}^{\prime} e^{j k_{0} z} d S
$$

Multiplying and dividing the right-hand side of the above equation by the quantity $2 Z_{0} \ln (b / a)$ it is readily found that:

$$
\frac{1}{2 \pi q b} \iint_{S_{A}} J_{m s \varphi}^{\prime} e^{j k_{0} z} d S=-2 \frac{R_{0} \alpha^{+}}{q Z_{0} / 2 \pi}
$$

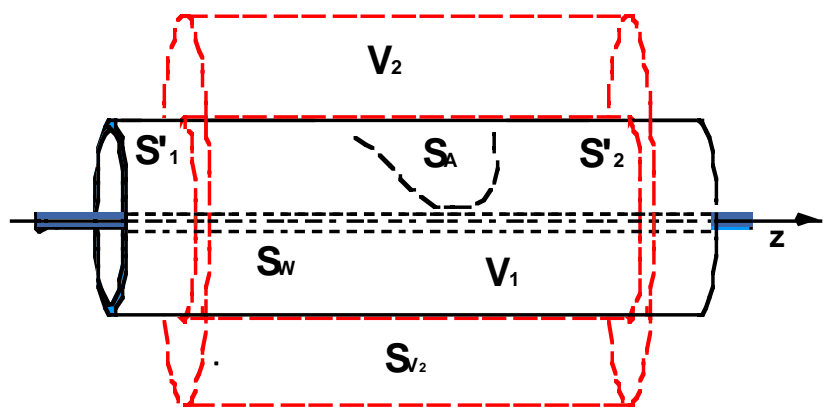

Figure 1: Geometry of the structure.

Regarding the coaxial structure as a two-port network, it is possible to express the right-hand side of the equation (16) by means of the circuital parameters of such device. In fact, if the output line is terminated in a matched load and on the input side we put a generator, matched to the line, such as to produce the incident wave provided by (9), the transmission parameter of the S-matrix is given by:

$S_{2,1}^{D U T}=\left.\frac{V_{2}^{-}}{V_{1}^{+}}\right|_{V_{2}^{+}=0}=\frac{\left(q Z_{0} / 2 \pi+\alpha^{+}\right)}{q Z_{0} e^{-j k_{0} z_{1}^{\prime}} / 2 \pi} e^{-j k_{0} z_{2}^{\prime}}=\left(1+\frac{\alpha^{+}}{q Z_{0} / 2 \pi}\right) e^{-j k_{0} l}$

where $l=z_{2}^{\prime}-z_{1}^{\prime}$.

For a portion of unperturbed coaxial line of length $l$, we have:

$$
S_{2,1}^{R E F}=e^{-j k_{0} l}
$$

Then, using (16) and (18), we obtain:

$$
Z(\omega)=-2 \frac{R_{0} \alpha^{+}}{q Z_{0} / 2 \pi}=2 R_{0}\left(\frac{S_{2,1}^{R E F}-S_{2,1}^{D U T}}{S_{2,1}^{R E F}}\right)
$$

\section{IMPEDANCE FOR A SMALL BUT FINITE RADIUS OF THE WIRE}

Our aim is now to account for the effect of the central conductor with a small but finite radius.

Applying twice the Reciprocity Theorem in relation to the volumes $V_{2}$ and $V_{1}$ (fig.1), with the equivalent currents $\left(-\vec{J}_{m s}^{\prime},-\vec{J}_{m s}\right)$ in $V_{2}$ and $\left(\vec{J}_{m s}^{\prime}, \vec{J}_{m s}\right)$ in $V_{1}$ as fields sources, and using the fields given by eqs.(8) and (11) we get the equations:

$$
\begin{array}{r}
Z(\omega)+Z^{\prime *}(\omega)=\frac{1}{q^{2}}\left[\iint_{S_{V_{2}}}\left(\vec{E}^{\prime *} \times \vec{H}+\vec{E} \times \vec{H}^{\prime *}\right) \cdot d \vec{S}\right. \\
\left.\quad-\iint_{S_{A}} \vec{H}_{s}^{\prime *} \cdot \vec{J}_{m s} d S-\iint_{S_{A}} \vec{H}_{s} \cdot \vec{J}_{m s}^{, *} d S\right]
\end{array}
$$




$$
\iint_{S_{A}} \vec{H}_{s}^{* *} \cdot \vec{J}_{m s} d S+\iint_{S_{A}} \vec{H}_{S} \cdot \vec{J}_{m s}^{\prime *} d S=-\iint_{S_{W}} \hat{i}_{r} \times \vec{H}_{s}^{\prime *} \cdot \vec{E}_{S} d S
$$

where $Z^{\prime}(\omega)$ is the impedance in presence of the wire and $S_{W}$ is the whole wire surface.

For very small wire radius, we may put $\vec{E}_{s}(r=a, \varphi, z) \approx \vec{E}_{s}(r=0, z)$ and, assuming the aperture smaller than the wavelenght, the equations (19) and (20) give:

$Z(\omega)+Z^{\prime *}(\omega)-\frac{Z^{\prime *}(\omega) Z(\omega)}{2 R_{0}}=\frac{1}{q^{2}} \iint_{S_{V_{2}}}\left(\vec{E}^{\prime *} \times \vec{H}+\vec{E} \times \vec{H}^{\prime *}\right) d \vec{S}$

In this expression the perturbed terms are unknown and depend on the wire thickness. Assuming that for small radius, there is a little difference between the perturbed and unperturbed fields, we will consider two perturbative approaches:

\subsection{Substitution $(\vec{E}, \vec{H}) \rightarrow\left(\vec{E}^{\prime}, \vec{H}^{\prime}\right)$}

We obtain:

$$
Z(\omega)=\frac{4 R_{0}^{2}}{4 R_{0}^{2}-\left|Z^{\prime}(\omega)\right|^{2}}\left[Z^{\prime}(\omega)-\frac{\left|Z^{\prime}(\omega)\right|^{2}}{2 R_{0}}\right]
$$

which can be expressed by means of the transmission $S$ parameters:

$$
\begin{aligned}
Z(\omega)= & \frac{2 R_{0}}{1-\left|S_{2,1}^{R E F}-S_{2,1}^{D U T}\right|^{2}} . \\
& {\left[\frac{S_{2,1}^{R E F}-S_{2,1}^{D U T}}{S_{2,1}^{R E F}}-\left|S_{2,1}^{R E F}-S_{2,1}^{D U T}\right|^{2}\right] }
\end{aligned}
$$

We observe that when the wire radius $a$ tends to zero the above equation approaches the Sands and Rees formula.

\subsection{Substitution $\left(\vec{E}^{\prime}, \vec{H}^{\prime}\right) \rightarrow(\vec{E}, \vec{H})$}

We get:

$$
Z(\omega)=\frac{2 R_{0}}{2 R_{0}-Z^{\prime *}(\omega)}\left[Z^{\prime}(\omega)-\frac{\left|Z^{\prime}(\omega)\right|^{2}}{R_{0}}\right]
$$

which can be express by means of the transmission $S$ parameters as:

$$
\begin{aligned}
Z(\omega)= & \frac{2 R_{0} S_{2,1}^{D U T} S_{2,1}^{R E F^{*}}}{\left|S_{2,1}^{D U T}\right|^{2}} . \\
& {\left[\frac{S_{2,1}^{R E F}-S_{2,1}^{D U T}}{S_{2,1}^{R E F}}-2\left|S_{2,1}^{R E F}-S_{2,1}^{D U T}\right|^{2}\right] }
\end{aligned}
$$

We observe that also this relation tends to the Sands and Rees formula when the wire radius approaches zero. Neglecting the second order term in the square brackets, it can be rewritten as:

$$
Z(\omega)=2 R_{0} \frac{S_{2,1}^{R E F}-S_{2,1}^{D U T}}{S_{2,1}^{D U T}}\left(\frac{q Z_{0} / 2 \pi+\alpha^{+}}{q Z_{0} / 2 \pi+\alpha^{+^{*}}}\right)
$$

which becomes similar to the Hahn and Pedersen formula for a small radius.

\section{COMPARISON WITH EXPERIMENTAL DATA}

We have performed measurements with the coaxial wire method on a device similar to fig. 1 with four narrow slots as aperture [10]. The results are shown in fig.2, where the curves corresponding to: a) ref. [4]; b) ref. [5]; c) eq. (23); d) eq. (25). We notice that there are no visible difference between a) and c), and b) and d).

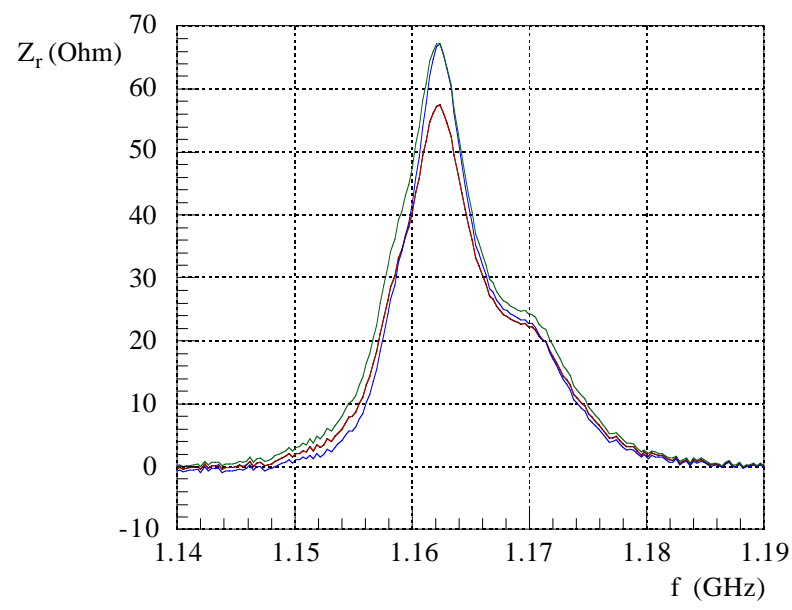

Figure 2: Real part of impedance calculated using the expressions given by: a) $\bullet$ ref. [4]; b) • ref. [5]; c) • eq. $(23)$; d) • eq. (25).

\section{REFERENCES}

[1] M. Sands and J. Rees, SLAC-Report PEP-95, (1974).

[2] G. Lambertson, unpublished, (1987).

[3] F. Caspers, Proc. of the Workshop on impedance and current limitations, (ERSF-Grenoble, France, octr 1988), CERN PS/88-59.

[4] L. Palumbo and V. G. Vaccaro, LNF-89/035(P), (1989).

[5] H Hahn, F. Pedersen, BNL 50870, Particle Accelerators and High-Voltage Machines TID4500, (1978).

[6] L. S. Walling et al., NIM A 281, pp. 433-447, (1989).

[7] V. G. Vaccaro, INFN/TC-94/023, (1994).

[8] R. L. Gluckstern, Physical Rev. A, vol.46, pp. 1106-1115, (1992).

[9] S. S. Kurennoy, Report SSLC-636, (1983).

[10] A. Argan, " L' impedenza longitudinale negli acceleratori: teoria e misura", laurea thesis 1998. 